\title{
Strip Steel Surface Defects Recognition Based on SOCP Optimized Multiple Kernel RVM
}

\author{
Hou Jingzhong, Xia Kewen (D), Yang Fan, and Zu Baokai $i$ \\ School of Electronics \& Information Engineering, Hebei University of Technology, Tianjin 300401, China \\ Correspondence should be addressed to Xia Kewen; kwxia@hebut.edu.cn
}

Received 10 September 2017; Accepted 8 February 2018; Published 29 March 2018

Academic Editor: Ningde Jin

Copyright (C) 2018 Hou Jingzhong et al. This is an open access article distributed under the Creative Commons Attribution License, which permits unrestricted use, distribution, and reproduction in any medium, provided the original work is properly cited.

\begin{abstract}
Strip steel surface defect recognition is a pattern recognition problem with wide applications. Previous works on strip surface defect recognition mainly focus on feature selection and dimension reduction. There are also approaches on real-time systems that mainly exploit the autocorrection within some given picture. However, the instances cannot be used in practical applications because of a bad recognition rate and low efficiency. In this paper, we study the intelligent algorithm of strip steel surface defect recognition, where the goal is to improve the accuracy and save running time. This problem is very important in various applications, especially the process testing of steel manufacturing. We propose an approach called the second-order cone programming (SOCP) optimized multiple kernel relevance vector machine (MKRVM), which can recognize strip surface defects much better than other methods. The method includes the model parameter estimation, training, and optimization of the model based on SOCP and the classification test. We compare our approach with existing methods on strip surface defect recognition. The results demonstrate that our proposed approach can improve the recognition accuracy and reduce the time costs of the strip surface defect.
\end{abstract}

\section{Introduction}

There are different types of defects in strip steel production due to various causes. These defects affect the appearances of the products and cause concentrated stress and weak spots. Moreover, these defects will decrease the product's properties in varying degrees, such as corrosion resistance and wear resistance [1]. Therefore, it is very important for enterprises to strengthen the inspection and control of strip surface defects and improve the strip surface quality [2]. If we want to improve the strip surface quality, detection and classification problems must be solved for the strip surface quality. These years, there are many study methods on strip surface defect recognition, including the exhaustive method and branch and bound method. For example, there are also the support vector machine (SVM) method, the principal component analysis method, the neural networks method, the magnetic flux leakage detection method, the infrared detection method, and the eddy current testing method. However, some of these methods may damage the topological structures of the data and some may get local optimal solutions. The Particle Swarm Optimization algorithm is an efficient optimization method, but its complex genetic operations make it difficult to meet the expected recognition speed and accuracy. These algorithms cannot take into account defects recognition.

Therefore, further study is necessary to improve the accuracy of strip surface defect recognition. Compared with the SVM, the RVM compensates for the disadvantages of the SVM [3] that include the lack of sparsity, the lack of probabilistic factors, the limitation by Mercer, and more. To improve the universal applicability of the RVM algorithm, multiple kernel learning (MKL) has been applied instead of one specific kernel function. However, the training time of the multiple kernel RVM (MKRVM) is long because of the inverse matrix problem in the algorithm. If the training sample and iteration numbers are increased, the training process becomes very slow.

Second-order cone programming (SOCP) $[4,5]$ is a convex optimization in which a linear function is minimized. Linear programs and convex quadratic programs can all be formulated as SOCP optimizations. Therefore, a SOCP optimized MKRVM algorithm is proposed, which optimizes the MKRVM parameters using the SOCP model. Through the optimized SOCP, the classification accuracy and time 
costs of the MKRVM algorithm can be largely improved. First, typical strip defect images are collected for model training and building the data set. Second, the simplified information and the key indicators that reflect the defects are obtained after strip defect images are addressed. This process can reduce the learning time of the MKRVM. Third, the model of the MKRVM based SOCP is trained, which is used to recognize the defect images data set. To improve the recognition accuracy, the design and construction of the multikernel RVM classifier are completed, and multikernel learning is transformed into the SOCP problem. Finally, the proposed SOCP-MKRVM [6] model is used for classification and identification. The effectiveness of the proposed method is verified.

\section{Principle of Identification Method}

2.1. Traditional RVM Method. Assuming that the training set is $\left\{x_{i}, y_{i}\right\}(i=1,2, \ldots, m)$, the target value $y_{n}$ and the input value $x_{n}$ are independently distributed samples. The output of the RVM $[7,8]$ can be expressed as

$$
y_{j}=\sum_{i=1}^{m} w_{i} K\left(X_{j}, X_{i}\right)+w_{0}+\varepsilon_{j} .
$$

Here, $K($,$) is the kernel function. The Gauss kernel$ function is used as the kernel function in this paper:

$$
K\left(x_{1}, x_{2}\right)=\exp \left(-\frac{\left\|x_{1}-x_{2}\right\|^{2}}{2 \sigma_{1}^{2}}\right) .
$$

$w$ is the weight vector of the model. $\varepsilon_{n}$ is the additional noise that satisfies the Gaussian distribution:

$$
\varepsilon_{n} \sim N\left(0, \sigma^{2}\right)
$$

Deriving from formula (1) and formula (3), we get

$$
p\left(y \mid w, \sigma^{2}\right)=N\left(\Phi w, \sigma^{2}\right)
$$

In the formula, $\Phi$ is a designed $m \times(m+1)$ order matrix consisting of kernel functions such that $\Phi=\left[\phi\left(x_{1}\right)\right.$, $\left.\phi\left(x_{2}\right), \ldots, \phi\left(x_{m}\right)\right]^{T}$ and $\phi\left(x_{i}\right)=\left[1, k\left(x_{i}, x_{1}\right), k\left(x_{i}, x_{2}\right), \ldots\right.$, $k\left(x_{i}, x_{m}\right)$ ]. The ARD Gaussian prior probability distribution of the defined weights is

$$
p(w \mid \alpha)=\prod_{i=0}^{m} N\left(w_{i} \mid 0, \alpha_{i}^{-1}\right) .
$$

Here, $\alpha$ is the $m+1$ dimension vector composed of super parameters that control the distribution of $w, A=$ $\operatorname{diag}\left(\alpha_{0}, \alpha_{1}, \ldots, \alpha_{m}\right)$.

According to Bayesian theory, the posterior probability distribution of $w$ can be decomposed as

$$
p\left(w \mid y, \alpha, \sigma^{2}\right)=N(u, \Sigma)
$$

where

$$
\begin{aligned}
p\left(y \mid \alpha, \sigma^{2}\right) & =\int p\left(y \mid w, \sigma^{2}\right) p(w \mid \alpha) d w . \\
\Sigma & =\left(\sigma^{-2} \Phi^{T} \Phi+A\right)^{-1} \\
u & =\sigma^{-2} \Sigma \Phi^{T} y
\end{aligned}
$$

$u$ and $\Sigma$ are the mean vector and covariance matrix of $w$. It can be seen from formula (9) that the parameter $\sigma^{2}$ and the super parameter $\alpha$ need to be estimated. The posterior probability distribution of the parameter $\sigma^{2}$ and the super parameter $\alpha$ is obtained according to the Bayesian theory as follows:

$$
p\left(\alpha, \sigma^{2} \mid y\right)=\frac{p\left(y \mid \alpha, \sigma^{2}\right) p\left(\alpha, \sigma^{2}\right)}{p(y)} .
$$

The estimation of the parameter $\sigma^{2}$ and the super parameter $\alpha$ is the calculation of the maximum value of the posterior probability distribution. It can be seen from (10) that the value of the posterior probability distribution of the two parameters is mainly determined by the likelihood function $p\left(y \mid \alpha, \sigma^{2}\right)$ :

$$
p\left(y \mid \alpha, \sigma^{2}\right)=N(0, C)
$$

Here, $C=\sigma^{2} I+\Phi A^{-1} \Phi^{T}$.

The maximum likelihood estimation method is used to calculate the maximum value of (11). The parameter $\sigma^{2}$ and the super parameter $\alpha$ are obtained as

$$
\begin{aligned}
\alpha_{i}^{\text {new }} & =\frac{1-\alpha_{i} \sum_{i i}}{u_{i}^{2}} \\
\sigma^{2 \text { new }} & =\frac{\|y-\Phi u\|^{2}}{m-\sum_{i=0}^{m}\left(1-\alpha_{i} \Sigma_{i i}\right)} .
\end{aligned}
$$

In the iterative operation, much of the super parameter $\alpha_{i}$ tends to infinity. $w$ obtained from formula (9) corresponds to the trend of zero. Therefore, you can make a small number of sample points work to achieve the RVM's sparse effect [9], and these sample points are of relevance to the vector machine.

2.2. Multiple Kernel Learning (MKL) Algorithms. To improve the universal applicability of the RVM algorithm, MKL has been applied instead of one specific kernel function:

$$
K_{\mu}\left(\mathbf{x}_{i}, \mathbf{x}_{j}\right)=f_{\mu}\left(\left\{K_{m}\left(\mathbf{x}_{i}, \mathbf{x}_{j}\right)\right\}_{m=1}^{M} \mid \mu\right),
$$

where $K_{\mu}$ is the multikernel function. The multiple kernel $K_{\mu}$ can be obtained by function $f_{\mu}: R^{D} \rightarrow R^{P}$ and by combining $M$ different $K_{m} \cdot \mu$ is the proportion parameter of the kernel. 
The kernel weights are regarded as a regularization item $r(\mu)$, which is taken as $(1 / 2)(\mu-1 / M)^{T}(\mu-1 / M)$. Thus, the objective function and gradient function can transform into

$$
\begin{array}{r}
J(\mu)=\frac{1}{2} \sum_{i=1}^{n} \sum_{j=1}^{n} y_{i} y_{j} \alpha_{i} \alpha_{j} K_{\mu}\left(\mathbf{x}_{i}, \mathbf{x}_{j}\right)-\sum_{i=1}^{n} \alpha_{i}-r(\mu), \\
\frac{\partial J(\mu)}{\partial \mu_{m}}=\frac{1}{2} \sum_{i=1}^{n} \sum_{j=1}^{n} y_{i} y_{j} \alpha_{i} \alpha_{j} \frac{\partial K_{\mu}\left(\mathbf{x}_{i}, \mathbf{x}_{j}\right)}{\partial \mu_{m}}-\frac{\partial r(\mu)}{\partial \mu_{m}}
\end{array}
$$

The kernel combined function is

$$
\begin{aligned}
K_{\mu}\left(\mathbf{x}_{i}, \mathbf{x}_{j}\right) & =\prod_{m=1}^{M} \exp \left(-\mu_{m}\left(\mathbf{x}_{i}^{m}-\mathbf{x}_{j}^{m}\right)^{2}\right) \\
& =\exp \left(\sum_{m=1}^{M}-\mu_{m}\left(\mathbf{x}_{i}^{m}-\mathbf{x}_{j}^{m}\right)^{2}\right) .
\end{aligned}
$$

2.3. Second-Order Cone Programming (SOCP). The above classification algorithm is based on the edge likelihood estimation method. However, this algorithm has an extensive matrix inversion process, and thus the training time is longer. To solve this problem, the MKRVM method based on the SOCP algorithm is proposed in this paper.

The standard form of the second-order cone programming is

$$
\min \left\{\sum_{i=1}^{n} c_{i}^{T} x_{i}: \sum_{i=1}^{n} A_{i} x_{i}=b, x_{i} \in K_{i}, i=1, \ldots, n\right\} .
$$

Here, $x_{i} \in K_{i}, i=1, \ldots, n$, is the variable. $b \in R^{m}$ and $A_{i} \in R^{m \times k}, c_{i} \in R^{k_{i}}, i=1, \ldots, n$, is the known quantity. $K_{i}, i=1, \ldots, n$, is the second-order cone of dimension $k_{i}$. Thus

$$
K_{i}:=\left\{x_{i}=\left(x_{i 0}, x_{i 1}\right) \in R \times R^{k_{i}-1}: x_{i 0}-\left\|x_{i 1}\right\| \geq 0\right\} .
$$

In this, $\|\cdot\|$ is the Euclidean norm of the vectors. It is easy to know that $K_{i}$ is self-dual and

$$
K_{i}^{0}:=\left\{x_{i}=\left(x_{i 0}, x_{i 1}\right) \in R \times R^{k_{i}-1}: x_{i 0}-\left\|x_{i 1}\right\|>0\right\} .
$$

For the MKRVM, the focus of its operations is the iterative computation of $\alpha_{i}$. The purpose of the SOCP is to change the original solution and efficiently solve this parameter. The Gauss kernel function is a typical local kernel, and the polynomial kernel function is a typical global kernel. The combination of the two kernel functions can have the advantages of both local and global kernels. Let $x_{i}$ and $y_{i}$ be the sample points in the data space. The combination kernel function is

$$
K=\mu_{1} G_{1}\left(x_{i}, y_{j}\right)+\mu_{2} G_{2}\left(x_{i}, y_{j}\right)+\mu_{3} P\left(x_{i}, y_{j}\right) .
$$

Here,

$$
\begin{aligned}
G_{1}\left(x_{i}, y_{j}\right) & =\left(-\frac{\left\|x_{i}-y_{j}\right\|^{2}}{\theta_{1}^{2}}\right) ; \\
G_{2}\left(x_{i}, y_{j}\right) & =\left(-\frac{\left\|x_{i}-y_{j}\right\|^{2}}{\theta_{2}^{2}}\right) ; \\
P\left(x_{i}, y_{j}\right) & =\left[x_{i}\left(\frac{y_{j}}{\theta_{3}^{2}}\right)+1\right]^{2} .
\end{aligned}
$$

$\theta_{1}, \theta_{2}$, and $\theta_{3}$ are the kernel parameters obtained by nuclear correction. $\mu_{1}, \mu_{2}$, and $\mu_{3}$ are the undetermined combination coefficients.

In the multikernel functions, the kernel correction value of the kernel function $K$ is

$$
A(\beta)=A\left(S, K(\beta), y y^{T}\right)=\frac{\sum_{k} \beta_{k} y^{T} K_{k} y}{\sqrt[m]{\sum_{k 1} \beta_{k} \beta_{l}\left\langle K_{k}, K_{l}\right\rangle_{F}}}
$$

in which $S$ is the sample data.

Then, the process of obtaining the best correction value of the kernel function is transformed into the following planning problem:

$$
\begin{aligned}
\max _{\beta}: & A(\beta) \\
\text { subject to: } & 1 \geq \beta_{i} \geq 0 \\
& \sum_{j=1}^{M} \beta_{i}=1 .
\end{aligned}
$$

After calculating $\beta_{i}$ of each kernel function, the validity of the kernel parameter is judged according to the value of $\beta_{i}$. If $\beta_{i}$ approaches 0 , it means that the corresponding kernel parameter of $\beta_{i}$ is not suitable for use on this data set. If the value of $\beta_{i}$ is close to 1 , then the corresponding kernel parameter of $\beta_{i}$ is valid.

2.4. MKRVM Method Based on SOCP Algorithm. SOCP is used to optimize the MKRVM $[10,11]$, and $\mu_{1}, \mu_{2}$, and $\mu_{3}$ are the kernel work parameters that need to be optimized. The previous part of (21) is the reciprocal of a Euclidean paradigm, and the latter exponential function is a convex function. In this case, the optimization problem can be solved by being transformed into a standard SOCP problem [12]. In the SOCP form, the parameters are solved efficiently through the MKRVM training and the optimization process by using the SeDuMi and Yamlip toolboxes.

The SOCP-MKRVM algorithm, which mainly uses the MKRVM, calculates $\alpha_{i}$ by using the method of base vector filling, which efficiently calculates the multikernel parameters. The main algorithmic steps of the SOCP-MKRVM are as follows.

Step 1. Load the training samples and initialize the parameters of the MKRVM. 
TABLE 1: Comparison of the two methods.

\begin{tabular}{lcccc}
\hline Algorithm & Test error (\%) & Number of iterations & Computation time (s) & Number of RVM \\
\hline RVM & 8.6 & 446 & 2.7520 & 4 \\
SOCP-MKRVM & 8.1 & 255 & 0.5620 & 4 \\
\hline
\end{tabular}

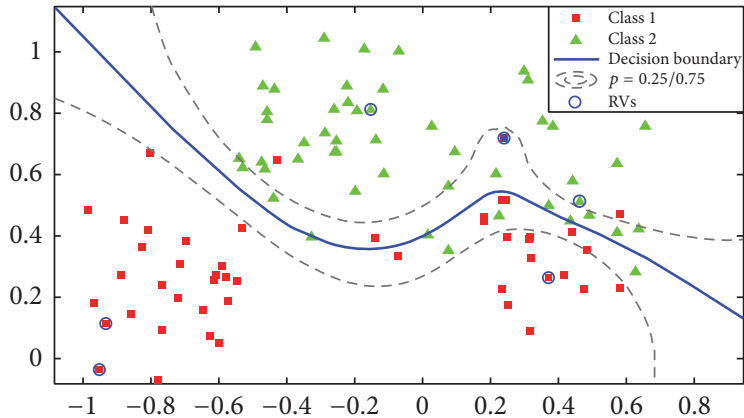

FIGURE 1: Pipley classification results of the RVM.

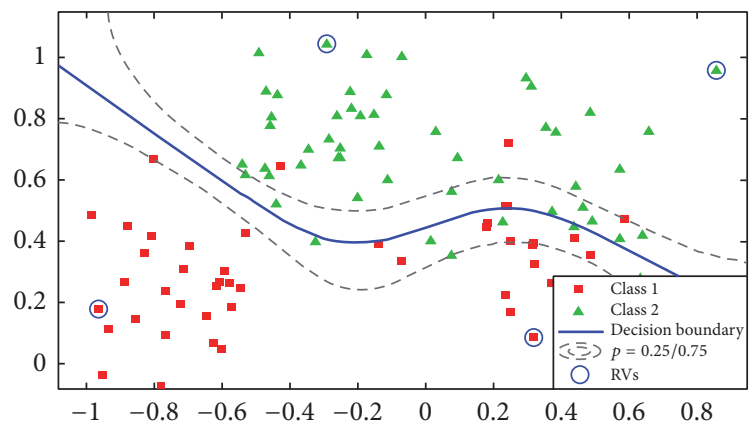

FIGURE 2: Pipley classification results of the SOCP-MKRVM.

Step 2. Construct a multikernel function as (19).

Step 3. Use formula (22) for the nuclear correction and solve the nuclear parameters.

Step 4. Put the unknown kernel working parameters into the SOCP and calculate it.

Step 5. Get the optimal values of the kernel function parameters and put them into the MKRVM model for classification.

2.5. Classic Example Simulation. In the MKRVM classification verification, Pipley's synthetic (a commonly used data set for classification) is used for the test. The data set is a two-dimensional classification sample set with 250 training samples and 1000 test samples. There is much Gaussian mixed noise in Pipley's synthetic data set. We use the edge likelihood estimation iteration and the SOCP optimization to classify the data by computer simulation, and the results are shown in Figures 1 and 2. The effect of the two methods is shown in Table 1.

As seen from Table 1, under the training and testing of the same data set, the number of RMV is reduced, the model is sparser and the running time is shortened after the SOCP optimizes the MKRVM. In addition, in the classification test of the Pipley data set, the test error in the optimized program is smaller, which indicates that the classification accuracy is improved.

\section{Experiment Simulation and Result Analysis}

3.1. Identification System Design. The whole detection algorithm is divided into the offline processing part and the online detection and recognition part. The offline processing part includes training sample set selection, image defect pretreatment, SOCP-MKRVM model training, and the preservation of the trained SOCP-MKRVM model for online defect recognition. The online detection and recognition part is divided into real-time processing section and on-time processing section. For the real-time processing part, first we obtain a binary image by preprocessing the image. Then, label the binary image by judging whether the binary image has a defect. If a defect appears in the image, we locate the defective region and save the single defect area as a bitmap. After that, the defect bitmaps are put into on-time processing section for processing. If there is no defect in the image, process the next image frame. The algorithm of this section is simple, but it requires processing every frame of an image, which increases the amount of data to be processed. The on-time processing section extracts the features of a single defective image region obtained from the real-time processing section. Then, load the selected features into the SOCP-MKRVM model for classification and recognition. Simultaneously, load the defect location information and the category information into the database in real time. The algorithm is complex, but the amount of data processing is small after the real-time processing section screens it. Thus, the two sections' times could be linked. Finally, fast and accurate defect detection is realized.

The algorithm training process flow chart and the strip defect recognition process flow chart are shown in Figures 3 and 4.

3.2. Application Environment and Sample Selection. The experimental environment is as follows. The PC hardware consisted of an Intel Core i5 $7602.8 \mathrm{GHz}$ CPU with $2 \mathrm{~GB}$ of RAM. The software platform was comprised of the Windows 7 Operating system and MATLAB 7.0 simulation software.

In the strip production process, the continuous casting billet, rolling equipment, processing technology, and more result in different kinds of surface defects. These defects include abrasions, scar, scratches, bonding, roller marks, needle, holes, epidermal stratification, pitting, welds, inclusions, cracks, frown lines, scales, stains, burrs, bubbles, and more. The SOCP optimized MKRVM technology can be used to model and identify the strip surface defects and then 
TABLE 2: Table of defect feature parameters.

\begin{tabular}{|c|c|c|c|c|c|c|c|c|}
\hline & Perimeter & Centroid & Rectangularity & Roundness & Defect area & Average value & Mean square error & PSNR \\
\hline Scratches & 6752511.4677 & 9563 & $2.2573 e-005$ & 1.3081 & 23547 & 202.1134 & 17.3348 & 40.2710 \\
\hline Abrasions & 8059327.7211 & 12491.5 & $5.1263 e-005$ & 1.2885 & 21564.5 & 126.7753 & 12.5693 & 40.0457 \\
\hline Scar & 6336816.5311 & 12988.5 & $7.0503 e-005$ & 1.3047 & 5309.375 & 99.2674 & 25.6033 & 40.1967 \\
\hline Roller marks & 4230693.2402 & $81.5 \quad 63.5$ & $4.167 e-005$ & 1.2719 & 20172.75 & 146.5667 & 10.0764 & 40.2564 \\
\hline Holes & 5535146.4921 & $82.5 \quad 81.5$ & $2.2391 e-005$ & 1.2545 & 22112.75 & 147.6829 & 26.3304 & 40.1245 \\
\hline Edge crack & 4684413.5541 & $76.5 \quad 93.5$ & $3.1132 e-005$ & 1.2679 & 6652.125 & 117.1682 & 19.1387 & 40.1197 \\
\hline Surface upwarping & 9993410.5839 & 143.5109 .5 & $4.9846 e-005$ & 1.3733 & 20552.625 & 113.3258 & 29.1838 & 40.0771 \\
\hline Pitting & 7861938.1007 & 10683.5 & $1.5379 e-005$ & 1.2752 & 34749.125 & 159.3857 & 13.4814 & 40.0777 \\
\hline
\end{tabular}

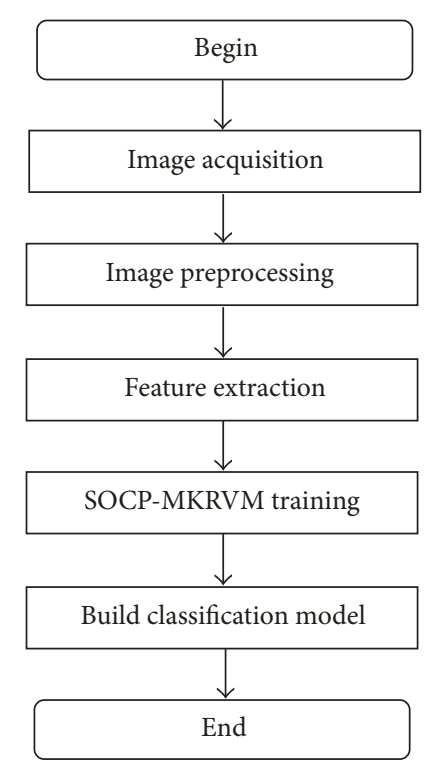

FIGURE 3: SOCP-MKRVM model training flow chart.

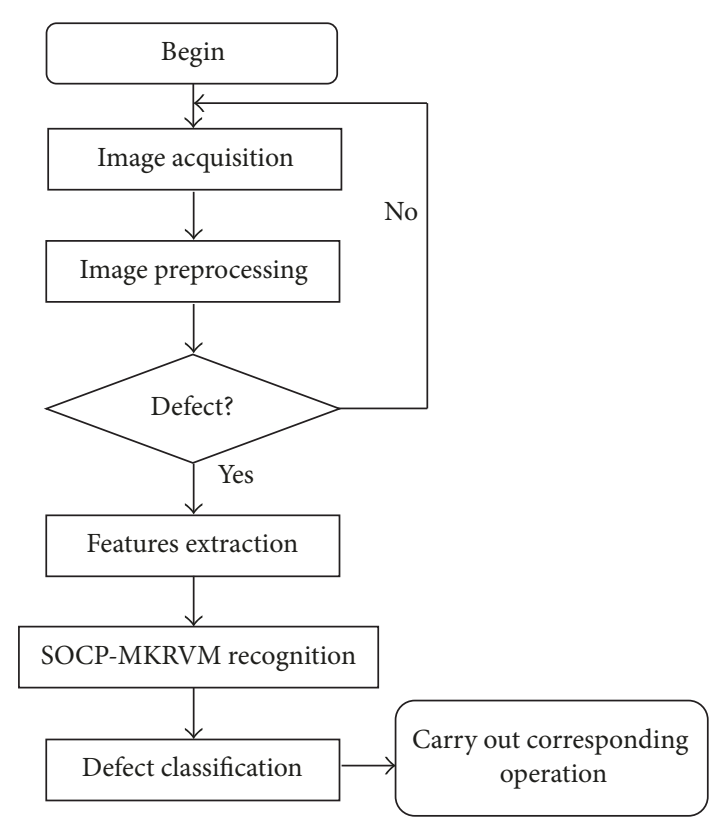

FIgURE 4: Flow chart of strip defect detection. correspondingly analyze the cause of the defects. Finally, the solution to eliminate the defects is proposed. In this experiment, the strip defect images are required from a steel factory. We choose the most common 8 kinds of defects as the research objects: scratches, abrasions, scars, roller marks, holes, edge cracks, and surface upwarping and pitting, as shown in Figures 5(a)-5(h), respectively.

3.3. Feature Parameter Extraction. The data set formed from parts of the required images is used in image preprocessing. In the recognition model training, three types of features are used, which include grayscale (mean value, mean square error, peak signal-to-noise ratio (PSNR), and the gray histogram), morphology (area, perimeter, and center of gravity), and texture (rectangular degree, roundness, and edge defects). The trained model is applied in the real-time recognition of defect images. Extract the feature information parameters, which are shown in Table 2. The gray histogram extracted from the defect images are shown in Figures $6(\mathrm{a})-6(\mathrm{~h})$ and the edge information of the defect images is shown in Figures $7(\mathrm{a})-7(\mathrm{~h})$.

Due to the use of the second-order cone programming method to optimize the parameters of the relevant vector machine, the time consumption of the SOCP-MKRVM algorithm in this paper is longer than the traditional RVM algorithm. However, the sample training modeling process is an offline process, and the real-time defect detection classification has no effect.

According to the above method, we extract the features of multiple images to establish the defect data set and then extract more factual information to build a highdimensional matrix. We capture numerous images to establish the database and constitute a matrix with 10-dimensional features as the training set, as shown in Table 3.

3.4. Results and Analysis. We simulate 8 respective types of common defects using the SOCP-MKRVM algorithm. There are 100 defect images for each defect. The testing samples and the defect recognition results are shown in Table 4. After that we simulate the 8 types of common defects with the traditional RVM algorithm and the traditional SVM algorithm; the results are shown in Table 4.

As shown in Table 4, the classification recognition accuracy of the SOCP-MKRVM algorithm is very high, and some 


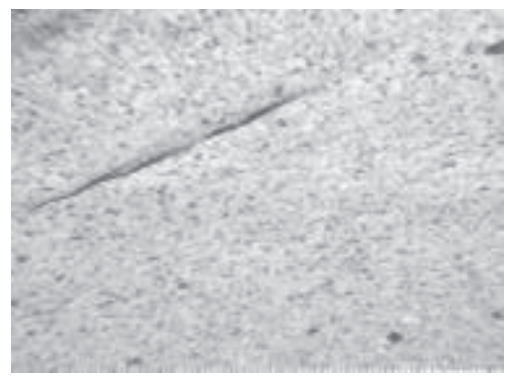

(a) Scratches

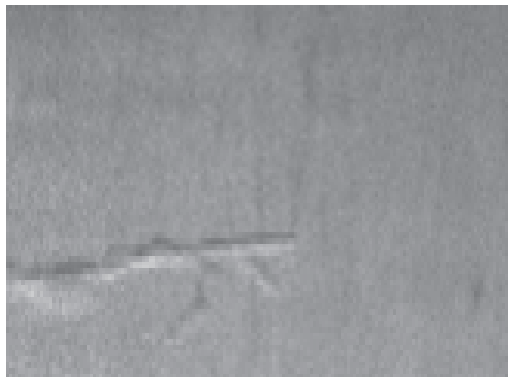

(d) Roller marks

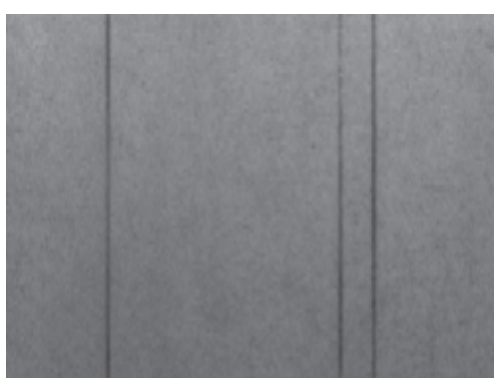

(b) Abrasions

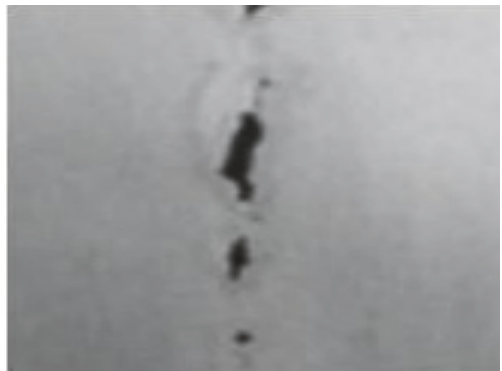

(e) Holes

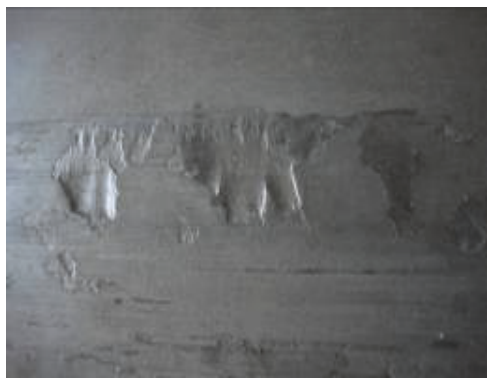

(c) Scars

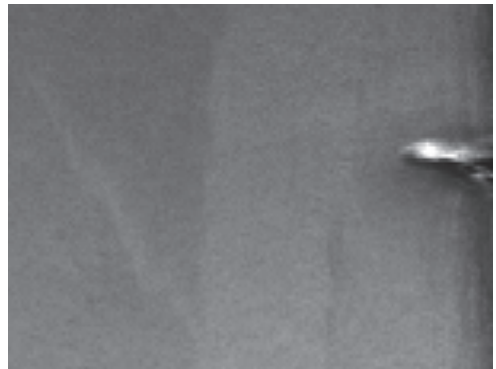

(f) Edge cracks

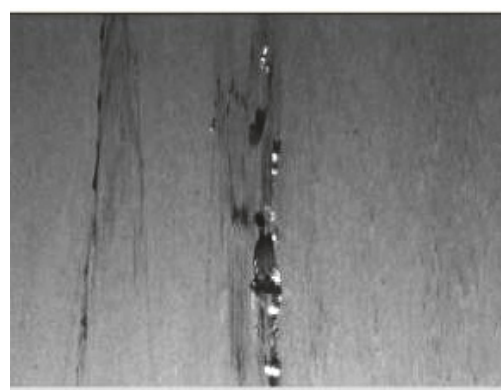

(g) Surface upwarping

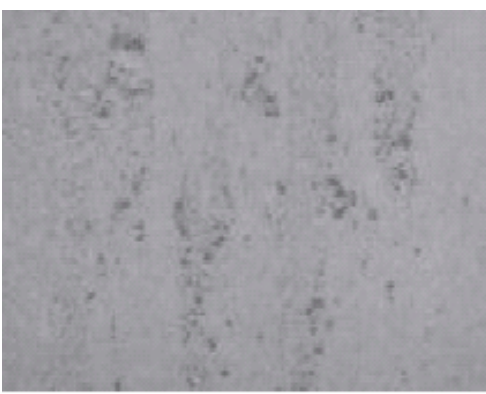

(h) Pitting

FIGURE 5: Strip steel defect images.

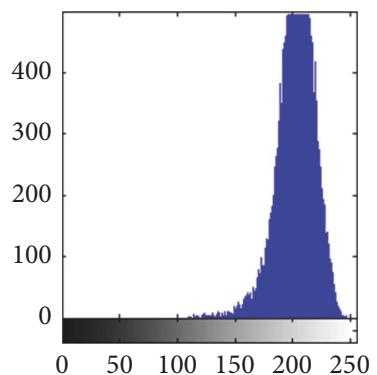

(a) Scratches

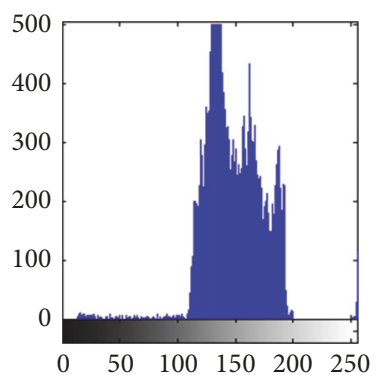

(e) Holes

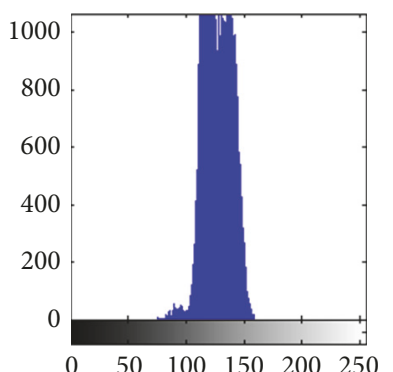

(b) Abrasions

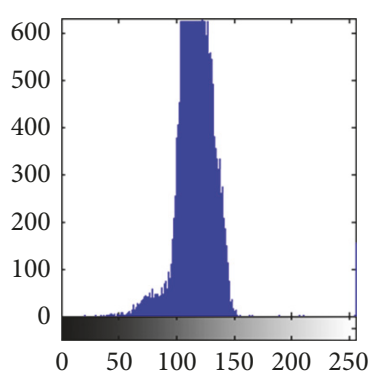

(f) Edge cracks

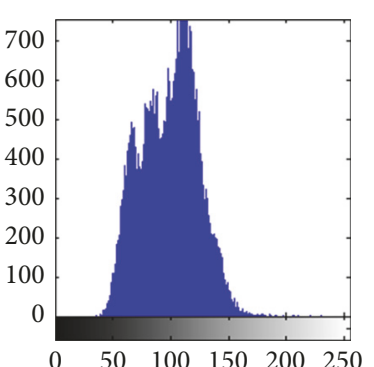

(c) Scars

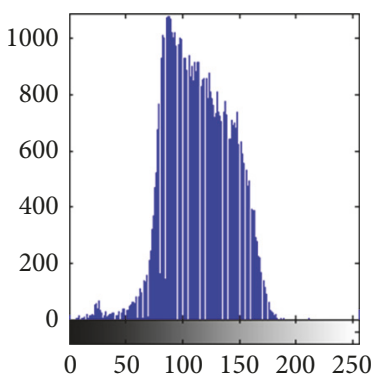

(g) Surface upwarping

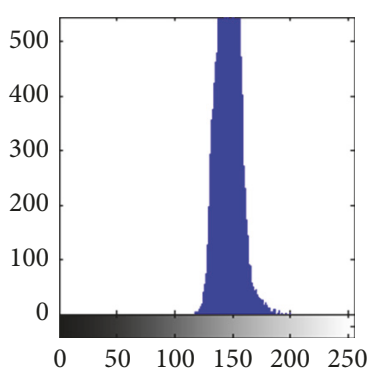

(d) Roller marks

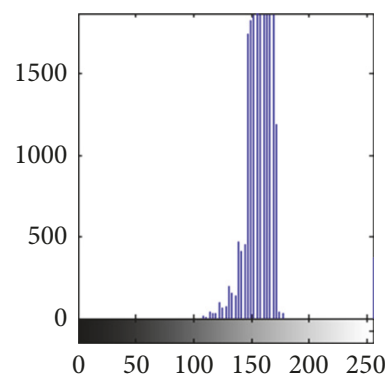

(h) Pitting

FIGURE 6: Gray histogram of defect images. 
TABLE 3: A matrix with 10-dimensional features.

\begin{tabular}{|c|c|c|c|c|c|c|c|c|c|}
\hline Perimeter & Centroid & Rectangularity & Roundness & Defect area & Average value & Mean square error & PSNR & Histogram & Edge \\
\hline$x_{i 1}$ & $x_{i 2}$ & $x_{i 3}$ & $x_{i 4}$ & $x_{i 5}$ & $x_{i 6}$ & $x_{i 7}$ & $x_{i 8}$ & $x_{i 9}$ & $x_{i 10}$ \\
\hline
\end{tabular}

TABLE 4: Defect recognition performance comparison of SOCP-MKRVM, RVM and SVM.

\begin{tabular}{|c|c|c|c|c|c|c|c|c|}
\hline Defect type & Scratches & Abrasions & Scar & Roller marks & Holes & Edge crack & Surface upwarping & Pitting \\
\hline Testing sample number & 100 & 100 & 100 & 100 & 100 & 100 & 100 & 100 \\
\hline Error number (SOCP-MKRVM) & 0 & 0 & 1 & 2 & 0 & 0 & 1 & 2 \\
\hline Recognition accuracy (SOCP-MKRVM) & $100 \%$ & $100 \%$ & $99 \%$ & $98 \%$ & $100 \%$ & $100 \%$ & $99 \%$ & $97 \%$ \\
\hline Time consuming (ms) (SOCP-MKRVM) & 6.42 & 6.27 & 7.87 & 7.87 & 5.87 & 6.02 & 7.37 & 7.28 \\
\hline Error number (RVM) & 1 & 0 & 4 & 5 & 0 & 0 & 3 & 2 \\
\hline Recognition accuracy (RVM) & $99 \%$ & $100 \%$ & $96 \%$ & $95 \%$ & $100 \%$ & $100 \%$ & $97 \%$ & $96 \%$ \\
\hline Time consuming (ms) (RVM) & 6.81 & 6.34 & 7.89 & 7.88 & 6.03 & 6.95 & 7.89 & 7.31 \\
\hline Error number (SVM) & 4 & 1 & 6 & 5 & 0 & 1 & 5 & 7 \\
\hline Recognition accuracy (SVM) & $96 \%$ & $99 \%$ & $94 \%$ & $95 \%$ & $100 \%$ & $99 \%$ & $95 \%$ & $93 \%$ \\
\hline Time consuming (ms) (SVM) & 6.95 & 7.34 & 8.29 & 9.18 & 6.73 & 8.05 & 8.32 & 8.16 \\
\hline
\end{tabular}

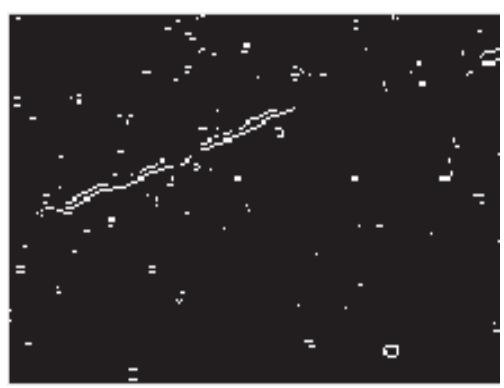

(a) Edge of scratches

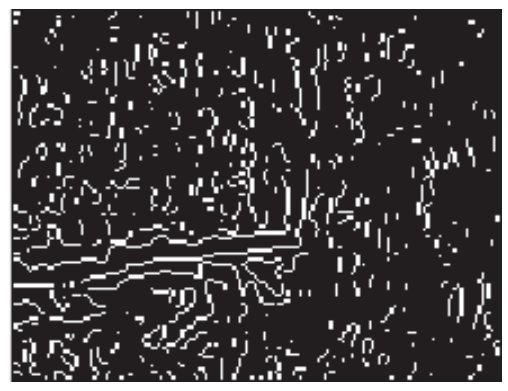

(d) Edge of roller marks

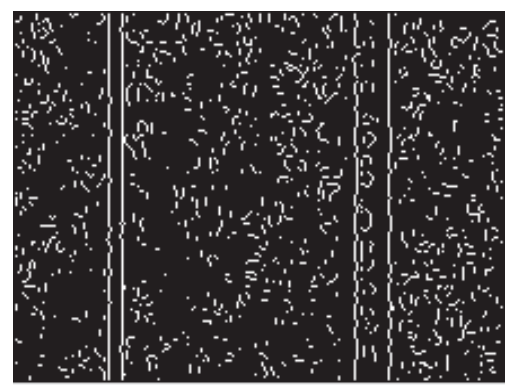

(b) Edge of abrasions

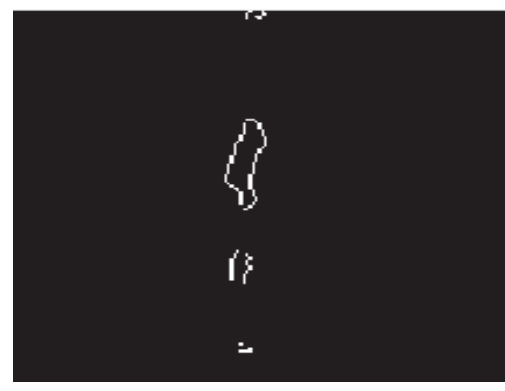

(e) Edge of holes

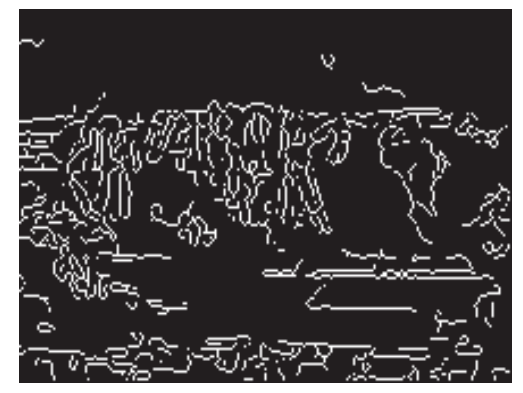

(c) Edge of scars

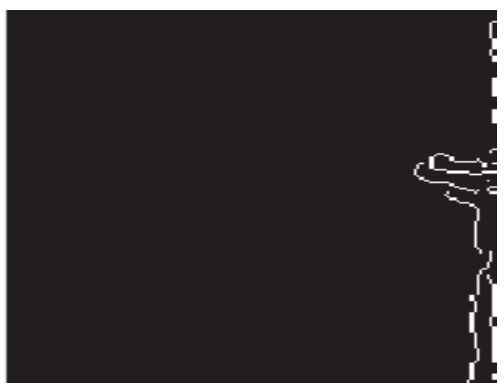

(f) Edge of edge cracks

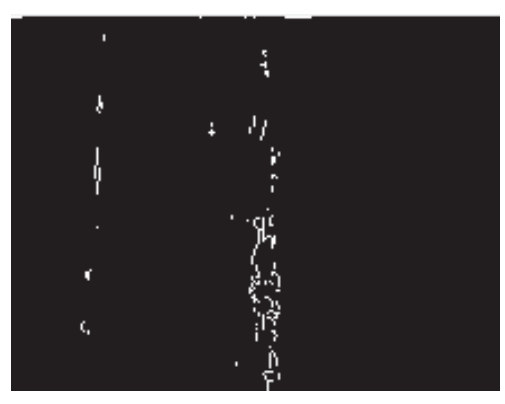

(g) Edge of surface upwarping

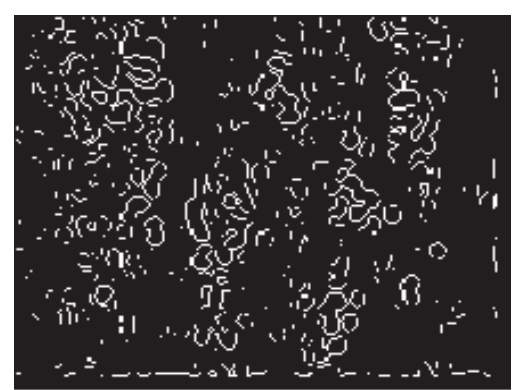

(h) Edge of pitting

FIGURE 7: Edge information of the defect images. 
of the defects have a $100 \%$ classification accuracy, such as scratches, abrasions, holes, and edge cracks. Only pitting has a slightly lower accuracy (97\%). Overall, the average recognition accuracy for the 8 types of defects is up to $99 \%$, which is very ideal.

The overall recognition accuracy of the traditional RVM algorithm and the traditional SVM algorithm is lower than that of the SOCP-MKRVM algorithm. Some types of defects also have very high recognition accuracy of RVM; however, three types (scars, roller marks, and surface upwarping) have relatively low recognition accuracy. The overall recognition accuracy of the traditional SVM algorithm is clearly lower than that of the SOCP-MKRVM algorithm. The average time consumption of the three algorithms is very short and is in milliseconds. For most of the overall detection results, the average time consumption of the SOCP-MKRVM algorithm is shorter than that of the two other algorithms. It is obvious that the SOCP-MKRVM algorithm is superior and more suitable than the traditional RVM algorithm and the traditional SVM algorithm in strip surface defect detection.

\section{Conclusion}

In this paper, we propose strip steel surface defect detection based on the SOCP optimized multiple kernel RVM. For the SOCP-MKRVM, the second-order cone programming method is used to optimize the relevance vector machine kernel function, which can make a more optimal selection for the kernel function and improve the classification accuracy and generalization ability. The SOCP-MKRVM algorithm has the advantages of high calculation accuracy and operation speed that effectively improves the working efficiency, reduces the intensity of workers, and has a very wide range of uses. The simulation experimental results show that the SOCP-MKRVM has significant advantages in accuracy and operation speed compared to the traditional RVM algorithm and the traditional SVM algorithm.

\section{Conflicts of Interest}

The authors declare that they have no conflicts of interest.

\section{References}

[1] K. Song and Y. Yan, "A noise robust method based on completed local binary patterns for hot-rolled steel strip surface defects," Applied Surface Science, vol. 285, pp. 858-864, 2013.

[2] H. G. Ramos, T. Rocha, J. Král, D. Pasadas, and A. L. Ribeiro, "An SVM approach with electromagnetic methods to assess metal plate thickness," Measurement, vol. 54, pp. 201-206, 2014.

[3] L. Ding and S. Liao, "KMA- $\alpha$ : a kernel matrix approximation algorithm for support vector machines," Computer Research and Development, vol. 49, no. 4, pp. 746-753, 2012.

[4] S. Maldonado and J. López, "Imbalanced data classification using second-order cone programming support vector machines," Pattern Recognition, vol. 47, no. 5, pp. 2070-2079, 2014.

[5] L. Julio and S. Maldonado, "Multi-class second-order cone programming support vector machines," Information Sciences, pp. 330-328, 2016.
[6] S. Maldonado, J. López, and M. Carrasco, "A second-order cone programming formulation for twin support vector machines," Applied Intelligence, vol. 45, no. 2, pp. 265-276, 2016.

[7] B. Zhang, W. Huang, L. Gong et al., "Computer vision detection of defective apples using automatic lightness correction and weighted RVM classifier," Journal of Food Engineering, vol. 146, pp. 143-151, 2015.

[8] V. Senthilkumar and M. Ezhilarasi, "An efficient multi-RVM classification-based ultrasound lung image retrieval approach," International Journal of Biomedical Engineering and Technology, vol. 22, no. 3, pp. 189-215, 2016.

[9] H. Hu, Y. Li, M. Liu, and W. Liang, "Classification of defects in steel strip surface based on multiclass support vector machine," Multimedia Tools and Applications, vol. 69, no. 1, pp. 199-216, 2014.

[10] C.-Y. Yeh, W.-P. Su, and S.-J. Lee, "An efficient multiplekernel learning for pattern classification," Expert Systems with Applications, vol. 40, no. 9, pp. 3491-3499, 2013.

[11] S. Maldonado and J. López, "Robust kernel-based multiclass support vector machines via second-order cone programming," Applied Intelligence, vol. 46, no. 4, pp. 983-992, 2017.

[12] S. K. Veeramani and E. Muthusamy, "Detection of abnormalities in ultrasound lung image using multi-level RVM classification," The Journal of Maternal-Fetal and Neonatal Medicine, vol. 29, no. 11, pp. 1844-1852, 2015. 


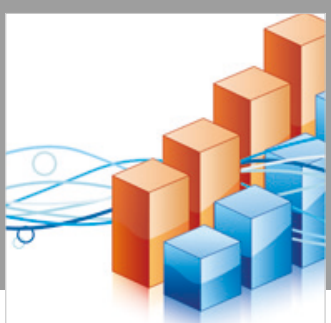

Advances in

Operations Research

\section{-n-m}
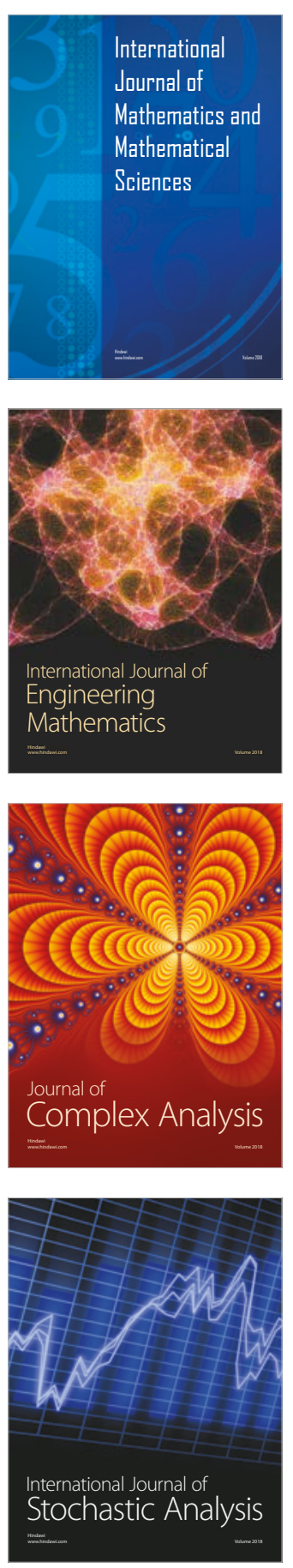
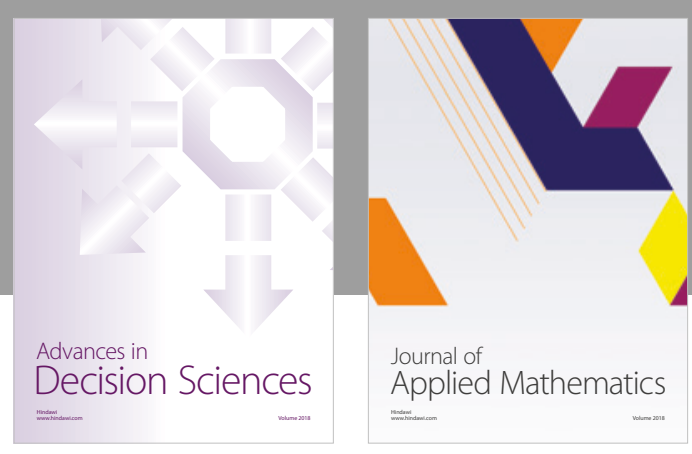

Journal of

Applied Mathematics
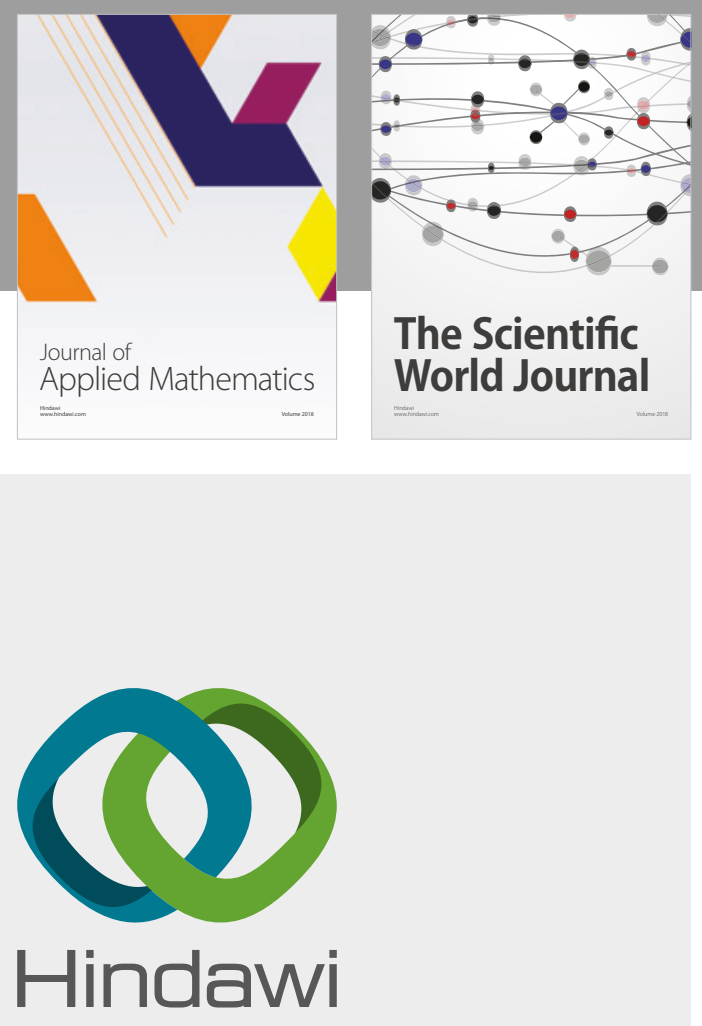

Submit your manuscripts at

www.hindawi.com

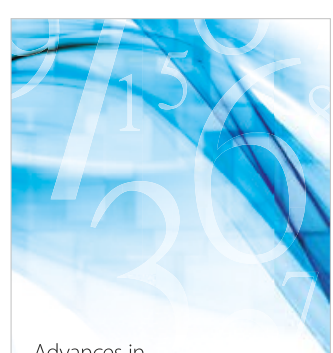

Advances in
Numerical Analysis
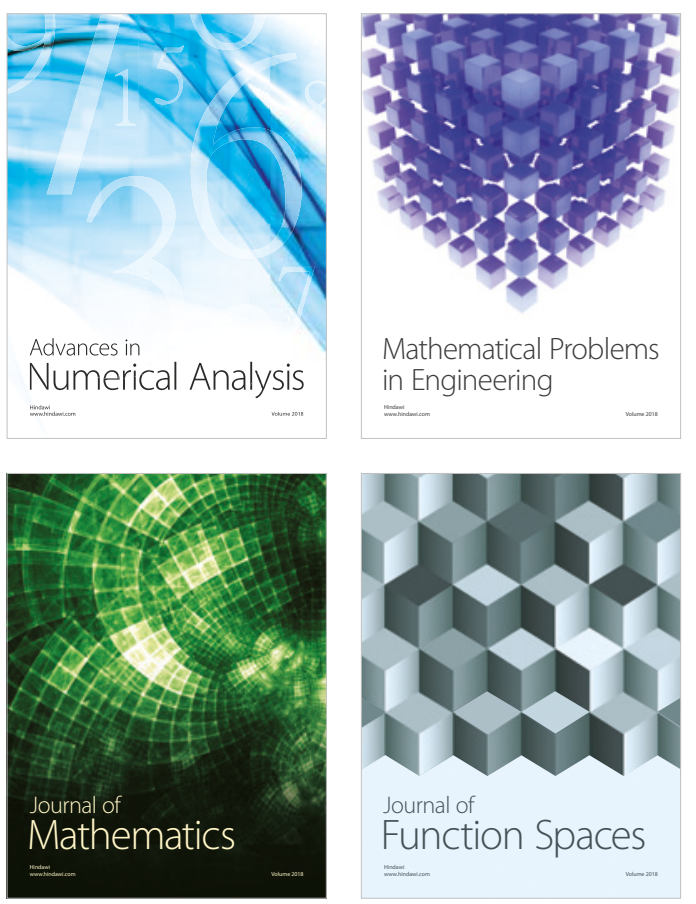

Mathematical Problems in Engineering

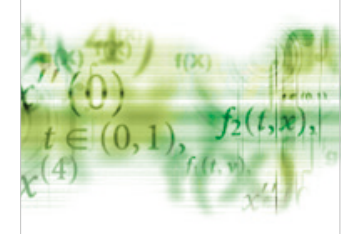

International Journal of

Differential Equations

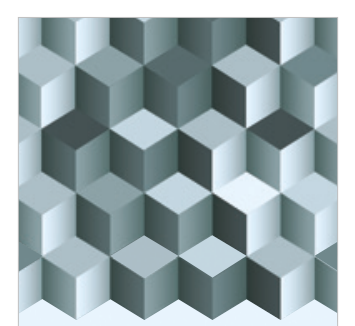

Journal of

Function Spaces

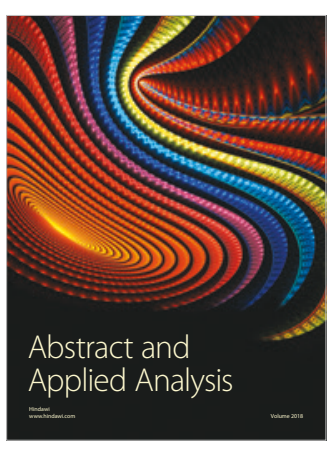

The Scientific

World Journal

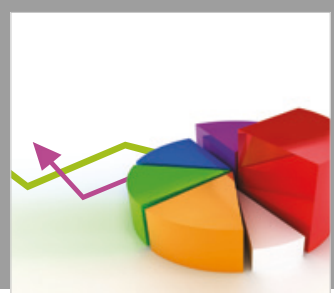

Journal of

Probability and Statistics
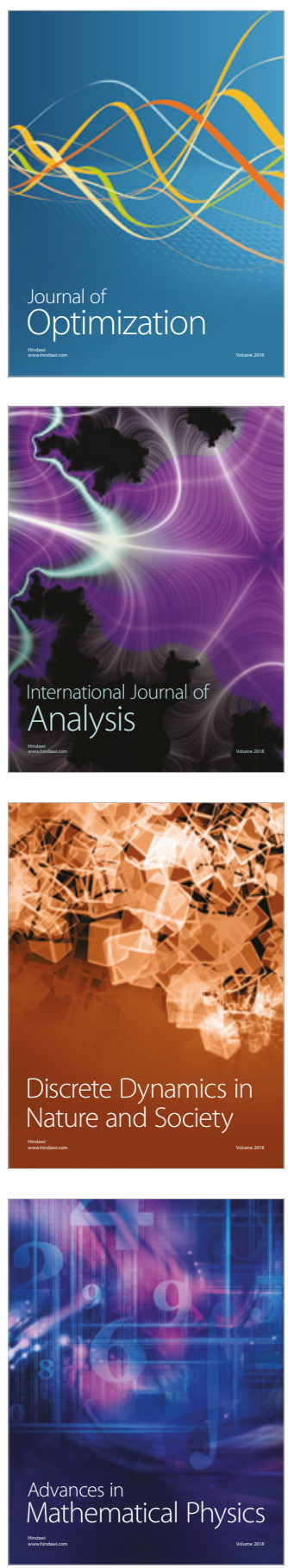